\title{
An Evacuation Decision Making Model for Firefighters in Ad Hoc Robot Network
}

\author{
Demin Li Jian Zou Kaikai Yue Hongyun Guan \\ College of Information Science and Technology \\ Donghua University \\ Shanghai 201620, China \\ deminli@dhu.edu.cn
}

\author{
Jiacun Wang \\ Department of Computer Science and Software \\ Engineering \\ Monmouth University \\ West Long Branch, NJ 07762, USA \\ jwang@monmouth.edu
}

\begin{abstract}
Evacuation for a firefighter in complex fire scene is challenge problem. In this paper, we discuss a firefighter's evacuation decision making model in ad hoc robot network on fire scene. Due to the dynamics on fire scene, we know that the sensed information in ad hoc robot network is also dynamically variance. So in this paper, we adapt dynamic decision method, Markov decision process, to model the firefighter's decision making process for evacuation from fire scene. In firefighting decision making process, we know that the critical problems are how to define action space and evaluate the transition law in Markov decision process. In this paper, we discuss those problems according to the triangular sensors situation in ad hoc robot network and describe a decision making model for a firefighter's evacuation the in the end.
\end{abstract}

Keywords- Ad hoc robot network, information fusion, directed graph, evacuation for a firefighter)

\section{InTRODUCTION (HEADING 1)}

Evacuation, especially for a firefighter in complex fire scene is a challenge problem. In this paper, we discuss how to evacuate for a firefighter in fire scene. On the complex fire scene, a firefighter's searching and rescuing victims is a dangerous process. Bashyal and Venayagamoorthy [1] let a human remotely control one of the robots in the swarm for searching. Hashimoto et al. [2] discussed a human being participating as a swarm member for search and rescue. Ulf Witkowski etc. [3] presented an ad-hoc network communication infrastructure for multi-robot systems in disaster scenarios. The main objective of the ad hoc network is to provide the team of robots and humans with robust communications links inside a building full of smoke. Thibault Kruse etc. [4] introduced a robot navigation approach that takes into account human-centered requirements and the collaborative nature of the interaction between the human and the robot. The paper only discussed the path planning problem of robots, but did not consider the human path planning problem. Penders etc. [5] presented the technology developed for a swarm of robots assisting a firefighter, and discussed robots just like nodes in ad hoc networks how to establish communication network and how to localize and map the fire scene. But the paper did not discuss how a firefighter to get and use the information about fire scene to navigate. In the paper [6,7], we use smoke density gradient and dynamic triangular network to establish directed graph, and discuss that firefighter how to select safe path to depart. Due to the dynamics on fire scene, we know that the sensed information in ad hoc robot network is also dynamically variance. So in this paper, we adapt dynamic decision method, Markov decision process, to model the firefighter's decision making process for firefighter's evacuation from fire scene.

The remaining of the paper is organized as follows. In section two, we discuss ad hoc robot network model, including dynamic triangulation method and communication protocol selection. In section three, for decision making use, we discuss Markov decision process related to firefighting situation. Using information fusion and Markov decision process, in section four, we describe information fusion based decision making for a firefighter's evacuation. We conclude in section five.

\section{AD Hoc RoBOt NeTwORK MODEL}

Fig. 1 in [8] shows a sketch of an environment, covered by the ad-hoc local network build by robots. Robots are represented as circles, and the communication links among them are indicated by dashed line segments. Two white circles represent the beacons positioned as the entrance to the site. Whereas other beacons can change their positions, these two positions might be preserved, as beacons at these positions can have several missions. They will provide communication between the swarm and the external facilities. The thicker dashed lines indicate the obstacles in the environment. The part of the environment with no visible obstacles represents a triangulation. The grey circles indicates the positions of the robots in the case if the environment had no obstacles, in which case an equilateral triangular grid would be preserved. Some of these positions are still possible, but not necessary, as the communication network can function without them. Some positions indicated by 'crossed' grey circles are simply impossible.

For the deployed ad hoc robot network, we discuss the routing protocol selection. Various routing protocols have been developed and proposed for ad hoc networks, which can be classified in reactive, proactive and hybrid protocols. Proactive protocols can offer low latencies as the routes are already available when needed. Destination-Sequenced 
Distance-Vector Routing (DSDV) is a typical example for a proactive routing algorithm [10]. The routing protocol is suitable for a network whose topology is not changeable frequently. Consider the robots' moving mode and networks' deploying mode, in this paper, we adopt the DSDV as a routing protocol in ad hoc robot network.

\section{MARKOV DECISION PROCESS FOR A FIREFIGHTER}

The firefighting decision process can be modeled a discrete-time Markov decision process (MDP).The basic definition [11,12] of a discrete-time MDP contains 5 components, (T, $\left.S, A_{s}, p_{\text {tran }}\left(s_{i} \mid s_{j}, a_{k_{l}}\right), r_{k}\left(s_{j}, a_{k_{l}}\right)\right)$, described using a standard notation. $T=1,2, \ldots, k, \ldots, N$ are the decision epochs, the set of points in time at which decisions are made (such as seconds or minutes in fire scene); $S$ is the state space with finite states, the set of all possible values of dynamic information relevant to the decision process. We define $S$ is a set of information (such as gas, temperature etc.) sensed by every robots. In other words, state $s_{j} \in S$ is information sensed by robot $j$; $A_{s}$ is the action space, the set of possible actions that the firefighter can take at state $s_{j}$ to get the exit. Where we suppose that $a_{k} \in A_{s}$ is an action at decision epoch $k$. The action $a_{k}$ represents a action of the firefighter who currently is nearby robot $j$ and is going to the neighbor location of robot $i ; p_{\text {tran }}\left(s_{i} \mid s_{j}, a_{k}\right)$ are the transition probabilities, which are conditional transition probability on the current state $s_{j}$ and action $a_{k}$ at the current decision epoch $k$ to the next state $s_{i}$; and $r_{k}\left(s_{j}, a_{k}\right)$ is the reward function, the immediate result of taking action $a_{k}$ at state $s_{j}$ and epoch $k$. As policy $a$ is a sequence of the decision rules or actions to be used at each decision epoch and defined as $a=\left(a_{1}, a_{2}, \ldots, a_{N-1}\right)$, the total reward of transformation from state $s_{j}$ to $s_{i}$ is $r\left(s_{j}, s_{i}\right)=\max _{a_{k} \in A_{s}}\left\{p_{\text {tran }}\left(s_{i} \mid s_{j}, a_{k}\right) r_{k}\left(s_{j}, a_{k}\right)\right\}$.In next section, we will combine ad hoc robot network model with Markov decision model to discuss how a firefighter make decision for his or her evacuation in detail.

\section{A DECISION MAKING MODEL FOR A FIREFIGHTER'S EVACUATION}

We know that evacuation is playing key role in search and rescue process. In this section, we discuss information fusion and a firefighter evacuation decision making based on dynamic triangular network. Because the deployment speed of dynamic triangular network may be slower than firefighter movement, while a firefighter is searching and rescuing, the robots can deploy the network by dynamic triangular network schema [8] as soon as possible. The evacuation decision making may be suitable for the situation when the firefighter has already finished rescuing task and ready to leave the fire scene. The authors in [13] informally clocked a guideline following exercise by experienced fire fighters: they progressed at crawling speed $12 \mathrm{~m}$ in about one minute. The amount of oxygen contained in the breathing apparatus suffices for about 20 minutes. Given the crawling speed, they can proceed about $240 \mathrm{~m}$ with a full tank. As they have to negotiate the 20 minutes of air between getting in and getting out, the maximum advance is only $120 \mathrm{~m}$. Due to the limited time for leave from the current location to the exit, the evacuation decision making is critical. In Fig. 2, a firefighter is going to leave the building from the exit after he or she has finished the rescuing task.

Due to the dynamics on fire scene, we know that the sensed information in ad hoc robot network is also dynamic variance. So in this paper, we adapt dynamic decision method, Markov decision process, to model the firefighter's decision making process for evacuation on fire scene. In firefighting decision making process, we know that the critical problem is how to define action space $A_{s}$, and evaluate the transition law $p_{\text {tran }}\left(s_{i} \mid s_{j}, a_{k}\right)$ in Markov decision process. In this section, we discuss those problems according to the triangular sensors situation in ad hoc robot network.

We only consider one-hop decision making problem, the two or more hop decision making problem can be operated similarly. For one-hop decision making process, the action space is $A_{s}=\{f l, f r, b l, b r\}$. Where $f l$ stands for the firefighter going forward to the left from one triangular vertex; fr stands for the firefighter going forward to the right from one triangular vertex; bl stands for the firefighter going backward to the left from one triangular vertex; $b r$ stands for the firefighter going backward to the right from one triangular vertex. We discuss how to get transition law $p_{\text {tran }}\left(s_{i} \mid s_{j}, a_{k}\right)$ for action $a_{k}$ at decision epoch $k$. In general situation, we consider $s_{j}$ has $m$ one-hop neighbors. For triangular sensor robot network in Fig.2, $m$ may be two, three or four. The firefighter currently is at the location of robot or sensor robot $j$. Consider $a_{k}$ may be two, three or four probable actions related to robot $j$ 's neighbors.

For firefighter or robot $j$ 's neighbors only have two sensor robots in Fig.3, a sub graph of Fig.2. In Fig.3, robot $j$ has two neighbors, robot $i_{1}$ and robot $i_{2}$. We use the temperature information $T\left(s_{i_{1}}\right), T\left(s_{i_{2}}\right)$ sensed by robot $i_{1}$ and robot $i_{2}$ to define the transition law $p_{\text {tran }}\left(s_{i} \mid s_{j}, a_{k}\right)$ as fellow in Fig.3.

$$
\begin{aligned}
& p_{\text {tran }}\left(s_{i_{1}} \mid s_{j}, a_{k}\right)=p_{\text {tran }}\left(s_{i_{1}} \mid s_{j}, a_{k}=f r\right) \\
& =\frac{1 / T\left(s_{i_{1}}\right)}{1 / T\left(s_{i_{1}}\right)+1 / T\left(s_{i_{2}}\right)}=\frac{T\left(s_{i_{2}}\right)}{T\left(s_{i_{1}}\right)+T\left(s_{i_{2}}\right)}
\end{aligned}
$$




$$
\begin{aligned}
& p_{\text {tran }}\left(s_{i_{2}} \mid s_{j}, a_{k}\right)=p_{\text {tran }}\left(s_{i_{2}} \mid s_{j}, a_{k}=f l\right) \\
& =\frac{1 / T\left(s_{i_{2}}\right)}{1 / T\left(s_{i_{1}}\right)+1 / T\left(s_{i_{2}}\right)}=\frac{T\left(s_{i_{1}}\right)}{T\left(s_{i_{1}}\right)+T\left(s_{i_{2}}\right)}
\end{aligned}
$$

For firefighter or robot $j$ 's neighbors have three sensor robots in Fig.4, a sub graph of Fig.2. In Fig.4, robot $j$ has three neighbors, robot $i_{1}$, robot $i_{2}$ and robot $i_{3}$. We use the temperature information $T\left(s_{i_{1}}\right), T\left(s_{i_{2}}\right), T\left(s_{i_{3}}\right)$ and sensed by robot $i_{1}$, robot $i_{2}$ and robot $i_{3}$ to define the transition law $p_{\text {tran }}\left(s_{i} \mid s_{j}, a_{k}\right)$ as fellow in Fig. 4

$$
\begin{aligned}
& p_{\text {tran }}\left(s_{i_{1}} \mid s_{j}, a_{k}\right)=p_{\text {tran }}\left(s_{i_{1}} \mid s_{j}, a_{k}=f r\right)=\frac{1 / T\left(s_{i_{1}}\right)}{1 / T\left(s_{i_{1}}\right)+1 / T\left(s_{i_{2}}\right)+1 / T\left(s_{i_{3}}\right)}= \\
& \frac{T\left(s_{i_{2}}\right) T\left(s_{i_{3}}\right)}{T\left(s_{i_{2}}\right) T\left(s_{i_{3}}\right)+T\left(s_{i_{1}}\right) T\left(s_{i_{3}}\right)+T\left(s_{i_{1}}\right) T\left(s_{i_{2}}\right)}
\end{aligned}
$$$$
p_{\text {tran }}\left(s_{i_{2}} \mid s_{j}, a_{k}\right)=p_{\text {tran }}\left(s_{i_{2}} \mid s_{j}, a_{k}=f l\right)=\frac{1 / T\left(s_{i_{2}}\right)}{1 / T\left(s_{i_{1}}\right)+1 / T\left(s_{i_{2}}\right)+1 / T\left(s_{i_{3}}\right)}
$$$$
=\frac{T\left(s_{i_{1}}\right) T\left(s_{i_{3}}\right)}{T\left(s_{i_{2}}\right) T\left(s_{i_{3}}\right)+T\left(s_{i_{1}}\right) T\left(s_{i_{3}}\right)+T\left(s_{i_{1}}\right) T\left(s_{i_{2}}\right)}
$$$$
p_{\text {tran }}\left(s_{i_{3}} \mid s_{j}, a_{k}\right)=p_{\text {tran }}\left(s_{i_{1}} \mid s_{j}, a_{k}=b r\right)=\frac{1 / T\left(s_{i_{3}}\right)}{1 / T\left(s_{i_{1}}\right)+1 / T\left(s_{i_{2}}\right)+1 / T\left(s_{i_{3}}\right)}
$$$$
=\frac{T\left(s_{i_{1}}\right) T\left(s_{i_{2}}\right)}{T\left(s_{i_{2}}\right) T\left(s_{i_{3}}\right)+T\left(s_{i_{1}}\right) T\left(s_{i_{3}}\right)+T\left(s_{i_{1}}\right) T\left(s_{i_{2}}\right)}
$$

Similarly, we can give transition law $p_{\text {tran }}\left(s_{i} \mid s_{j}, a_{k}\right)$ for firefighter or robot $j$ 's neighbors have four sensor robots. By now, we can give transition law $p_{\text {tran }}\left(s_{i} \mid s_{j}, a_{k}\right)$ for a firefighter with all possible situations.

In section 3, we know that the reward of transformation from state $s_{j}$ to $s_{i}$ is $r\left(s_{j}, s_{i}\right)=\max _{a_{k}}\left\{p_{\text {tran }}\left(s_{i} \mid s_{j}, a_{k}\right) r_{k}\left(s_{j}, a_{k}\right)\right\}$. So, for calculate the reward of transformation from state $s_{j}$ to $s_{i}$, we need to discuss the definition of $r_{k}\left(s_{i}, a_{k}\right)$. Consider it is difficult to position efficiently every sensor robots, so it is also difficult to calculate the distance between any two sensor robots. On fire scene, we need not to know the precise distance from the current location to the exit, we only need to know approximate distance in emergent situation. In triangular sensor robot network, if we know the communication hops (one hop means from one robot to its neighbor robot) from the current location to the exit, we could easily estimate the distance to the exit approximately. So, we can use the communication hops to definite $r_{k}\left(s_{i}, a_{k}\right)=\frac{h\left(s_{\text {ini }}, s_{\text {exit }}\right)}{h\left(s_{j}, s_{\text {exit }}\right)}$. Where $s_{i n i}$ is the firefighter's initial location state, $s_{\text {exit }}$ is the firefighter's exit location state, $s_{i}$ is the firefighter's current location state, $s_{j}$ is a state transformed from $s_{i}$ by take the action $a_{k}$. The function $h(x, y)$ means the communication hops from $x$ location state to $y$ location state.

Up to now, we can model the firefighter's decision problem as

$$
\begin{aligned}
& r\left(s_{i n i}, s_{\text {exit }}\right)=r\left(s_{i n i}, s_{i_{1}}\right)+r\left(s_{i_{1}}, s_{i_{2}}\right)+\ldots+r\left(s_{i_{N-1}}, s_{i_{N}}\right)+r\left(s_{i_{N}}, s_{\text {exit }}\right) \\
& =\max _{a_{1} \in A}\left\{p_{\text {tran }}\left(s_{i_{1}} \mid s_{\text {ini }}, a_{1}\right) r\left(s_{i n i}, a_{1}\right)\right\}+\max _{a_{2} \in A_{s}}\left\{p_{\text {tran }}\left(s_{i_{2}} \mid s_{i_{1}}, a_{2}\right) r\left(s_{i_{1}}, a_{2}\right)\right\}+\ldots+ \\
& \max _{a_{N} \in A_{s}}\left\{p_{\text {tran }}\left(s_{i_{N}} \mid s_{i_{N-1}}, a_{N}\right) r\left(s_{i_{N-1}}, a_{N}\right)\right\}+\max _{a_{N+1} \in A_{s}}\left\{p_{\text {tran }}\left(s_{\text {exit }} \mid s_{i_{N}}, a_{N+1}\right) r\left(s_{i_{N}}, a_{N+1}\right)\right\} \\
& =\max _{a} \sum_{k=0}^{N} r\left(s_{i_{k-1}}, s_{i_{k}}\right)
\end{aligned}
$$

The firefighter's decision making process is to search policy $a=\left(a_{1}, a_{2}, \ldots, a_{N+1}\right)$, a sequence of actions at each decision epoch.

\section{CONCLUSIONS}

In this paper, we give a firefighter's evacuation decision making model to solve the dynamic decision making problem on fire scene. For simplifying decision or action space, we only discuss one hop communication situation. For two or more hops communication situation, the decision space may be enlarged with exponentially, decision making complexity may be increased, and decision making responding time may be slowdown. Although we only discuss evacuation for fire scene, the method could be applied in other similar disaster situation, such as mine rescues, battlefield searches, etc.

In this paper, by primary experiment, we find that the one hop by hop decision making method may also waste time and lose the opportunity for evacuation on the fire scene. We think that some times on explicit fire spreading situation, we may use two or more hops decision making model, some times on vague fire spreading situation, we may use one hop decision making model, and we are dealing with this problem.

\section{ACKNOWLEDGMENT}

This work is partially supported by NSFC granted number 71171045; National ITER Plan grant number 2010GB108004; Shanghai Key Scientific Research Project under grant number $05 \mathrm{dz} 05036$

\section{REFERENCES}

[1] Shishir Bashyal and Ganesh Kumar Venayagamoorthy, "Human swarm interaction for radiation source search and localization", In IEEE Swarm Intelligence Symposium, pp. 1-8, 2008.

[2] Hiroshi Hashimoto, Sho Yokota, Akinori Sasaki, Yasuhiro Ohyama, Hiroyuki Kobayashi, "Cooperative interaction of walking human and distributed robot maintaining stability of swarm", In Proc. of 2nd IEEE/IES International Conference on Human System Interaction, pp.249-254, 2009

[3] Ulf Witkowski, Stefan Herbrechtsmeier, Andry Tanoto, Mohamed ElHabbal, Lyuba Alboul and Jacques Penders, "Self-optimizing human- 
robot systems for search and rescue in disaster scenarios”, In 7-th Heinz Nixdorf Symposium, pp. 315-329, Paderborn, Germany, 2008

[4] Thibault Kruse, Alexandra Kirsch, E. Akin Sisboty, Rachid Alami, "Exploiting Human Cooperation in Human-Centered Robot Navigation”, In The 19th IEEE International Symposium on Robot and Human Interactive Communication, RO-MAN , pp.1-6, 2010

[5] Penders, Jacques, Alboul, Lyuba, Witkowski, Ulf, Naghsh, Amir, Saezpons, Joan, Herbrechtsmeier, Stefan and Habbal, Mohamed El, "a robot swarm assisting a human fire-fighter", Advanced Robotics, Vol.25, No.1-2, pp. 93-117,2011

[6] Demin Li, Jie Zhou, Jiacun Wang, Yun Wang, Qianyi Zhang, Information Fusion and Path Selection for a Firefighter Based on Ad Hoc Robot Network, International Journal of Advancements in Computing Technology, accepted

[7] Qianyi Zhang, Demin Li, Yun Wang, Yanwei Chen, Yajuan An, Evacuation Path Selection for Firefighters Based on Dynamic Triangular Network Model, 2012 International Conference on Wireless Communications and Signal Processing, submitted

[8] Ulf Witkowski, Mohamed EI-Habbal, Stefan, Herbrechtsmeier, Andry Tanoto, Jacques Penders, Lyuba Alboul, Veysel Gazi, “Ad-hoc network communication infrastructure for multi-robot systems in

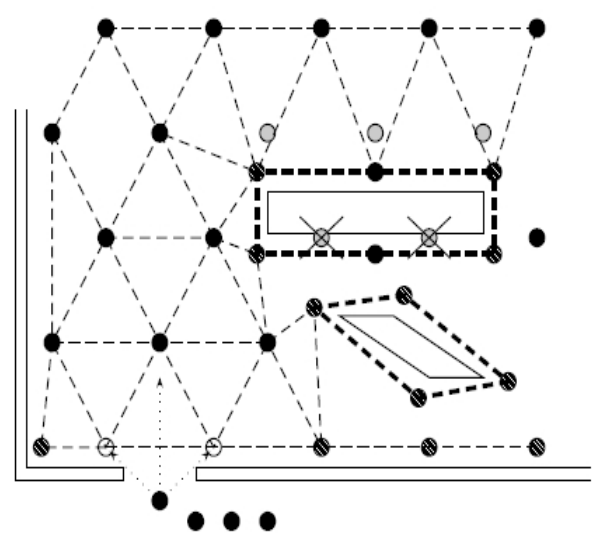

Fig. 1 Dynamic triangular network schema [8]

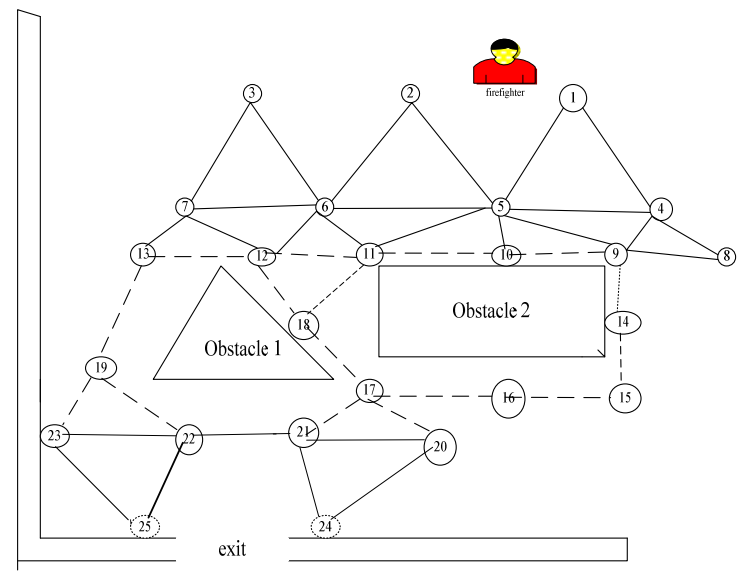

Fig. 2 Information Fusion and Escapable Graph [6] disaster scenarios”, In Proceedings of the EURON/IARP International Workshop on Robotics for Risky Interventions and Surveillance of the Environment, Benicassim, Spain ,pp. 1-8, 2008

[9] Sylviane Gentil, Jacky Montmain, "Hierarchical representation of complex systems for supporting human decision making”, Advanced Engineering Informatics, Vol. 18, No. 3, pp. 143-159 , July 2004

[10] Charles E. Perkins, Pravin Bhagwat, "Highly Dynamic DestinationSequenced Distance-Vector Routing (DSDV) for Mobile Computers”, In Proceeding of the SIGCOMM Conf. on Communications Architectures, Protocols and Applications, pp. 234-244, 1994

[11] Puterman ML., Markov Decision Processes, New York: John Wiley and Sons; 1994.

[12] Oguzhan Alagoz, Heather Hsu, Andrew J. Schaefer and Mark S. Roberts, Markov Decision Processes: A Tool for Sequential Decision Making under Uncertainty, Medical Decision Making, 30,474-483, 2010.

[13] Penders, Jacques, Alboul, Lyuba, Witkowski, Ulf, Naghsh, Amir, Saezpons, Joan, Herbrechtsmeier, Stefan and Habbal, Mohamed El, a robot swarm assisting a human fire-fighter. Advanced Robotics, 25 (1-2), 93-117,2011.

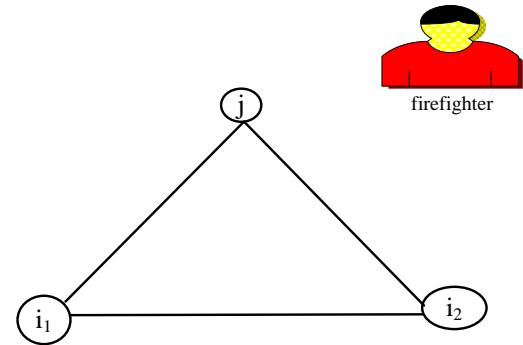

Fig. 3 Firefighter or robot $j$ 's neighbors only have two sensor robots

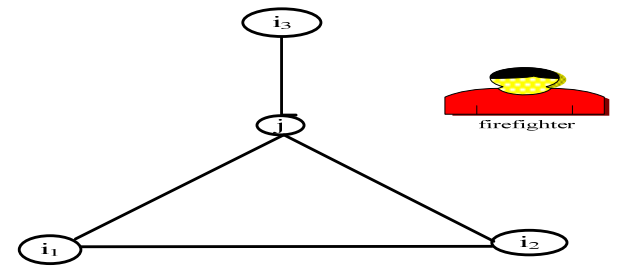

Fig. 4 Firefighter or robot $j$ 's neighbors have three sensor robots 